\title{
Ifosfamide, Carboplatin, and Etoposide (ICE) in Combination with Regional Hyperthermia as Salvage Therapy in Patients with Locally Advanced Nonmetastatic and Metastatic Soft-Tissue Sarcoma
}

\author{
Veit Bücklein (D), ${ }^{1}$ Christina Limmroth, ${ }^{2}$ Eric Kampmann, ${ }^{1}$ Gesa Schuebbe, ${ }^{1}$ Rolf Issels, \\ Falk Roeder, ${ }^{3}$ Martin Angele $\mathbb{D}^{4}{ }^{4}$ Hans Roland Dürr $\mathbb{D}^{5},{ }^{5}$ Thomas Knösel, ${ }^{6}$ \\ Sultan Abdel-Rahman, ${ }^{1}$ Dorit Di Gioia, ${ }^{1}$ and Lars H. Lindner $\mathbb{D}^{1}$
}

${ }^{1}$ Department of Medicine III, University Hospital, LMU Munich, Munich, Germany

${ }^{2}$ Medizinische Klinik Köln-Holweide, Cologne, Germany

${ }^{3}$ Department of Radiotherapy and Radio-Oncology, Landeskrankenhaus, Paracelsus Medical University Clinics, Salzburg, Austria

${ }^{4}$ Department of General, Visceral, and Transplant Surgery, University Hospital, LMU Munich, Munich, Germany

${ }^{5}$ Department of Orthopedics, University Hospital, LMU Munich, Munich, Germany

${ }^{6}$ Institute of Pathology, University Hospital, LMU Munich, Munich, Germany

Correspondence should be addressed to Lars H. Lindner; lars.lindner@med.uni-muenchen.de

Received 1 September 2019; Accepted 23 January 2020; Published 1 March 2020

Academic Editor: John D. Reith

Copyright (C) 2020 Veit Bücklein et al. This is an open access article distributed under the Creative Commons Attribution License, which permits unrestricted use, distribution, and reproduction in any medium, provided the original work is properly cited.

Patients with localized relapse of soft-tissue sarcoma (STS) after anthracycline-based chemotherapy have a dismal prognosis, particularly when surgery is not possible. To facilitate resection and improve long-term tumor control, we applied an intensified perioperative treatment consisting of ICE (ifosfamide $6 \mathrm{~g} / \mathrm{m}^{2}$, carboplatin $400 \mathrm{mg} / \mathrm{m}^{2}$, and etoposide $600 \mathrm{mg} / \mathrm{m}^{2}$ ) in combination with regional hyperthermia (RHT) to maximize local control. Here, we retrospectively evaluate the safety and efficacy of this strategy. Patients aged $\geq 18$ years with locally advanced high-risk STS, either with or without metastasis, treated with ICE + RHT after the failure of first-line anthracycline-based chemotherapy were included in this analysis. Radiographic response, toxicity, progression-free survival (PFS), and overall survival (OS) were assessed. Between 1996 and 2018, 213 sarcoma patients received ICE at our centre. Of these, 110 patients met the selection criteria (progressive disease, suitable high-grade STS histology, anthracycline pretreatment, RHT treatment) for this analysis. Fifty-four patients had locally advanced disease without metastases (LA-STS), and 56 patients had additional metastatic disease (M-STS). Disease control was achieved in 59\% of LA-STS patients and in $47 \%$ of M-STS patients. For LA-STS, $21 \%$ of the patients achieved radiographic response, facilitating resection in 4 patients (7\%), compared with $11 \%$ of the M-STS patients, facilitating resection in 5 patients (9\%). PFS was significantly longer in LA-STS than in M-STS (10 vs. 4 months, $p<0.0001$ ). Median OS was 26 months in LA-STS and 12 months in M-STS. Disease control was the only independent prognostic factor for improved OS in multivariate analysis. Toxicity was high with neutropenic fever occurring in $25 \%$ of the patients and three therapy-related deaths (3\%). ICE + RHT demonstrated activity in high-risk STS and facilitated resection in selected patients after anthracycline failure. Disease control was associated with improved OS. Based on the observed toxicities, the dose should be reduced to $75 \%$. 


\section{Introduction}

In the ongoing efforts to improve the outcome of patients suffering from soft-tissue sarcoma (STS), the management of local tumor relapse after intensive multimodal first-line therapy is rarely addressed [1]. For all STS patients, local relapse occurs in approximately $20 \%$ of patients [2-5], but these rates can be significantly higher in patients with insufficient resection margins and/or high-risk disease $[4,6,7]$. Patients with recurring STS have a disappointing prognosis $[4,8,9]$, illustrating the need for additional therapeutic options. This is particularly true for patients after failure of prior (neo)adjuvant chemo- and/or radiotherapy applied in high-risk situations.

Multimodal treatment including (neo)adjuvant anthracycline/ifosfamide-based chemotherapy in combination with regional hyperthermia (RHT) can improve progression-free and overall survival of STS patients with localized disease when compared with (neo)adjuvant chemotherapy alone [10]. Furthermore, response rates are doubled by the addition of RHT [7] and are higher than agents approved for use in the second-line treatment of STS (such as trabectedin, eribulin, or pazopanib) [11-14]. For patients with non- or borderlineresectable relapse of STS, intensification of local therapy by combining an anthracycline-free polychemotherapy with RHT might therefore be a promising therapeutic approach.

Here, for the first time, we report the outcomes of such an intensified multimodal therapeutic strategy, after anthracycline failure, in a larger cohort of patients. We retrospectively analyzed the safety, tolerability, and efficacy of a polychemotherapy consisting of ifosfamide, carboplatin, and etoposide (ICE) in combination with RHT in patients with relapsed/refractory STS. We included patients with local relapse or progression of their tumor after anthracycline-based chemotherapy. One subcohort encompassed patients without radiographic evidence of metastases (LASTS), whereas patients with predominant local relapse and concomitant metastatic disease were analyzed as a separate patient cohort (M-STS).

\section{Patients and Methods}

2.1. Patients. Patients aged $\geq 18$ years treated with ICE + RHT with a histological diagnosis of locally advanced high-risk STS, and progression or relapse of their disease after prior anthracycline-based chemotherapy, were included in this retrospective analysis. We divided the patients according to the presence or absence of metastases: patients with evidence of locally advanced STS without metastases (LA-STS) were analyzed separately from patients with metastatic disease (M-STS). All patients with metastatic disease, however, also suffered from local, RHT-targetable relapse of the STS. Evaluated histologic subentities for both populations encompassed adult-type STS according to the World Health Organization (WHO) classification of tumors [15]. Patients with gastrointestinal stromal tumors, clear cell sarcoma, angiosarcoma, and desmoplastic small round cell tumors were excluded due to differences in management and course of the disease.
Only patients with high-risk disease (defined as Fédération Nationale des Centres de Lutte Contre Le Cancer (FNCLCC) grade 2 or 3 , tumor diameter $\geq 5 \mathrm{~cm}$, located deep to the fascia) with radiographic evidence of disease progression were selected for the analysis. Patients who had not received an anthracycline-containing pretreatment or had not been treated with RHT were excluded. The analysis was approved by the local Institutional Review Board on Medical Ethics, and the Declaration of Helsinki was observed.

2.2. Treatment. ICE chemotherapy consisted of ifosfamide $\left(1,500 \mathrm{mg} / \mathrm{m}^{2}\right.$, days $\left.1-4\right)$, carboplatin $\left(100 \mathrm{mg} / \mathrm{m}^{2}\right.$, days $\left.1-4\right)$, and etoposide $\left(150 \mathrm{mg} / \mathrm{m}^{2}\right.$, days $\left.1-4\right)$. Treatment was repeated on day 28 until disease progression or for up to 8 cycles. Granulocyte-colony stimulating factor (G-CSF) support was mandatory. In case of insufficient blood count recovery, treatment was delayed. Dose reductions were applied if severe thrombocytopenia or neutropenic fever occurred, or in case of repeated treatment delays.

RHT, performed according to published quality and safety guidelines [16], was applied on days 1 and 3 or on days 1 and 4 of each ICE cycle. We used the BSD-2000 hyperthermia system (BSD Medical Corporation, Salt Lake City, UT, USA) to induce tumor temperatures of $40-43^{\circ} \mathrm{C}$ for 60 minutes. Ifosfamide was infused during RHT treatment. The RHT field was focused to the tumor area. For patients with metastatic disease, locoregional metastases (if present) were included in RHT field.

2.3. Imaging. Staging procedures encompassed computerized tomography (CT) scans for all patients, and magnetic resonance imaging (MRI) scans for patients with extremity tumors, and were usually performed after three, six, and eight cycles of treatment. After completion of treatment, radiographic reevaluation was performed every three months for two years, and every six months thereafter. Response was assessed according to Response Evaluation Criteria in Solid Tumors (RECIST) 1.1 [17]. Only subjects with measurable disease at the time of treatment initiation were included in the response assessment. For patients receiving tumor resection, radiographic response was given for the latest time point prior to resection.

2.4. Assessment of Hematological Toxicity and Infectious Complications. Due to the retrospective nature of the analysis, data on nonhematologic toxicity were limited. Frequencies of hematologic toxicity, however, were well documented, as grade IV toxicities (according to Common Terminology Criteria for Adverse Events [CTCAE] 4.03 criteria [18]) and infectious complications generally lead to dose reductions.

2.5. Statistical Analyses. To evaluate the efficacy of ICE + RHT, the progression-free rate (PFR) three and six months after the first administration of ICE + RHT was assessed in patients with metastatic disease (M-STS), with particular focus on patients who did not undergo tumor 
resection in the context of ICE + RHT. We focused on this subcohort to evaluate the efficacy of ICE + RHT, as these patients best resembled the patient population included in the publication of Glabbeke et al. [19], in which reference values for effective second-line treatment regimens for patients with STS have been determined.

Three- and six-month PFS were calculated for all patients (in both the LA-STS and M-STS cohorts) without clinical or radiographic evidence of progression who were alive three and six months after treatment initiation, respectively. PFS and OS were calculated according to the Kaplan-Meier method, and 95\% confidence intervals (CI) were estimated according to Greenwood's formula [20]. Patients without confirmed progression, relapse, or death after ICE + RHT treatment were censored at the time of the last assessment. Differences in survival were assessed by the stratified logrank test. A two-sided $p \leq 0.05$ was used to determine significance. Multivariate analyses were calculated using the Cox model. All statistical analyses were conducted using R (version 3.5.2) with the "Survival" package (Version 2.35) [21].

\section{Results and Discussion}

3.1. Patients' Characteristics. Between 1996 and 2018, 213 sarcoma patients were treated with ICE. Of these, 110 patients met the selection criteria and were included in the analysis. The LA-STS subcohort consisted of 54 patients, whereas 56 patients were included in the M-STS subgroup with metastatic disease (Supplementary Figure 1). Patients' characteristics are given in Table 1. Of note, ICE + RHT was applied as postoperative consolidation regimen after resection of relapse in $10(18.5 \%)$ LA-STS and 1 (1.8\%) M-STS patients, respectively (Table 2).

3.2. Treatment. In LA-STS patients, a median of four cycles of ICE chemotherapy were applied, together with a median of seven RHT treatments. In M-STS patients, the median of ICE cycles was also four, applied in combination with a median of six RHT treatments.

For all patients, particularly in the LA-STS subcohort of patients, (re-)resections and/or radiotherapeutic options were regularly reevaluated over the course of the therapy. Procedures shown in Table 2 encompass surgical treatments and radiotherapy applied in between cycles or after completion of ICE + RHT.

Details on ICE + RHT treatment duration, imaging results, and surgical procedures in LA-STS patients are depicted in Figure 1. Of note, tumor resection was performed in 31/54 (57\%) of LA-STS patients and in 15/56 (27\%) of M-STS patients. Complete resection with adequate margins (R0) was possible in $13 \%(7 / 54)$ of all patients without metastases and in $9 \%(5 / 56)$ of all patients with metastatic disease (including metastasectomy), respectively. R1 and R2 resections were performed in 10/54 (19\%) and 10/54 (19\%) LA-STS patients and in 4/56 (7\%) and 4/56 (7\%) M-STS patients, respectively. The resection margin status remained unclear (Rx) in $4 / 54$ (7\%) LA-STS and 2/56 (4\%) M-STS patients. In nine patients with radiographic response ( $8 \%$ of all treated patients), tumor resection was facilitated by ICE + RHT (four in LA-STS and five in M-STS patients, respectively).

3.3. Response to Treatment. For radiographic response, 40 patients could be evaluated in the LA-STS subcohort population, as ten patients had received ICE + RHT as postoperative consolidation regimen and had no measurable disease prior to ICE + RHT. No radiographic response assessment was performed in nine LA-STS patients due to clinical evidence of early disease progression in two patients, therapyrelated infectious deaths in two patients, and discontinuation of treatment without response assessment in five patients. In the M-STS subcohort, one patient was not evaluable for response due to resection (including metastasectomy) prior to ICE + RHT. In four patients, no radiographic response assessment was possible because of early clinical progression of the disease, and one patient discontinued treatment without response assessment. Results are given in Table 3. Objective responses were seen in 20\% (9/44) of LA-STS patients, compared with $11 \%(6 / 55)$ in M-STS patients. Disease control was obtained in 59\% (26/44) and 47\% (26/55) of the LA-STS and M-STS patients, respectively. Two patients with stable disease (SD) and three patients with initial partial remission (PR) progressed over continued treatment with ICE + RHT in the LA-STS cohort, whereas only one patient with initial PR achieved a (short-lasting) complete remission (CR) with further ICE + RHT therapy. In the M-STS subcohort, three patients with initial PR and eight patients with initial SD had progression of their STS with ongoing ICE + RHT treatment.

3.4. Survival. Median follow-up was 38 months (range $0-188$ months) for all censored patients $(n=12)$ in the LASTS subcohort at time of analysis, and median observation time of all analyzed LA-STS patients was 23 months. For patients with metastatic disease (M-STS), median follow-up of all censored patients $(n=6)$ and median observation time was 7 and 11 months, respectively.

3.4.1. Progression-Free Survival. Four of the 54 patients in the LA-STS subgroup (7\%) remained progression-free following multimodal treatment. Out of these four patients, ICE + RHT was applied as postoperative regimen in two patients and as preoperative regimen in two patients. Within the subgroup of patients with metastatic disease, progression of disease occurred in all 56 patients.

Progression-free rates (PFR) after 3 and 6 months are given in Table 4. Patients with metastatic disease without surgical treatment, the patient subgroup that best resembles the reference population from the original publication of Glabbeke et al. [19], had a PFR of $41 \%$ after three and $20 \%$ after six months, indicating activity of the regimen.

Median progression-free survival (PFS) was 10 months for all patients in the LA-STS population (95\% CI 8-11 months). It was significantly shorter for patients with metastatic disease (M-STS, 4 months [ $95 \%$ CI 2-5 months], $p<0.0001$, Figure 2). This was due to early progression of distant metastasis, as local PFS was not significantly different 
TABLE 1: Patients' characteristics and pretreatment. $p$ values, representing differences between patients with locally advanced disease and patients with metastatic disease, were assessed by Fisher's exact test for sex, progression vs. relapse, grading, and pretreatment procedures, by the chi-square test for anatomic site and histopathologic subtype, and by the unpaired $t$-test for age.

\begin{tabular}{|c|c|c|c|}
\hline & $\begin{array}{c}\text { Locally advanced disease } \\
\text { (LA-STS, } n=54) \\
\text { No. }(\%)\end{array}$ & $\begin{array}{l}\text { Metastatic disease } \\
\text { (M-STS, } n=56) \\
\text { No. }(\%)\end{array}$ & $p$ \\
\hline $\begin{array}{l}\text { Age } \\
\text { Median (range) } \\
<51 \text { years } \\
>50 \text { years }\end{array}$ & $\begin{array}{l}56(18-74) \text { years } \\
16(29.6) \\
38(70.4)\end{array}$ & $\begin{array}{l}51(21-77) \text { years } \\
27(48.2) \\
29(51.8)\end{array}$ & 0.23 \\
\hline $\begin{array}{l}\text { Sex } \\
\text { Male } \\
\text { Female } \\
\end{array}$ & $\begin{array}{ll}31 & (57.4) \\
23 & (42.6)\end{array}$ & $\begin{array}{l}26(46.4) \\
30(53.6)\end{array}$ & 0.26 \\
\hline $\begin{array}{l}\text { Progression vs. relapse } \\
\text { Progression of pre-existing lesion(s) } \\
\text { Relapse after disease-free interval } \\
\text { Median (range) duration of the disease-free interval }\end{array}$ & $\begin{array}{c}22(40.7) \\
32(59.3) \\
27(9-171) \text { months }\end{array}$ & $\begin{array}{c}37(66.1) \\
19(33.9) \\
14(2-115) \text { months }\end{array}$ & 0.01 \\
\hline $\begin{array}{l}\text { Anatomic site } \\
\text { Head and neck } \\
\text { Extremities and trunk wall } \\
\text { Extremities } \\
\text { Trunk wall } \\
\text { Trunk } \\
\text { Abdomen } \\
\text { Retroperitoneum } \\
\text { Pelvis }\end{array}$ & $\begin{aligned} 2 & (3.7) \\
23 & (42.6) \\
18 & (33.3) \\
5 & (9.3) \\
29 & (53.7) \\
10 & (18.5) \\
13 & (24.1) \\
6 & (11.1)\end{aligned}$ & $\begin{array}{cl}1 & (1.8) \\
30 & (53.6) \\
17 & (30.4) \\
13 & (23.2) \\
25 & (44.6) \\
15 & (26.8) \\
6 & (10.7) \\
4 & (7.1)\end{array}$ & 0.16 \\
\hline $\begin{array}{l}\text { Site of metastasis (multiple sites possible) } \\
\text { Lung } \\
\text { Liver } \\
\text { Nodal } \\
\text { Soft tissue } \\
\text { Osseous } \\
\text { Other } \\
\end{array}$ & & $\begin{array}{l}35(62.5) \\
13(23.2) \\
8(14.3) \\
7(12.5) \\
3(5.4) \\
8(14.3) \\
\end{array}$ & \\
\hline $\begin{array}{l}\text { Histopathologic subtype } \\
\text { Liposarcoma } \\
\text { Myxoid } \\
\text { Dedifferentiated } \\
\text { Leiomyosarcoma } \\
\text { Rhabdomyosarcoma } \\
\text { Undifferentiated pleomorphic sarcoma (UPS) } \\
\text { Synovial sarcoma } \\
\text { MPNST } \\
\text { Other }\end{array}$ & $\begin{aligned} 20 & (37.0) \\
2 & (3.7) \\
18 & (33.3) \\
8 & (14.8) \\
2 & (3.7) \\
11 & (20.4) \\
1 & (1.9) \\
5 & (9.2) \\
7 & (13.0)\end{aligned}$ & $\begin{array}{c}6(10.7) \\
0(0.0) \\
6(10.7) \\
16(28.6) \\
4(7.1) \\
14(25.0) \\
3(5.4) \\
4(7.1) \\
9(16.1)\end{array}$ & 0.051 \\
\hline $\begin{array}{l}\text { FNCLCC grading } \\
\text { Grade } 2 \\
\text { Grade } 3 \\
\end{array}$ & $\begin{array}{l}22(40.7) \\
32(59.3) \\
\end{array}$ & $\begin{array}{l}17(30.4) \\
39(69.6) \\
\end{array}$ & 0.32 \\
\hline $\begin{array}{l}\text { Pretreatment } \\
1 \text { regimen } \\
>1 \text { regimen } \\
\text { Anthracycline-containing } \\
\text { Ifosfamide-containing } \\
\text { Surgery } \\
\text { Radiotherapy }\end{array}$ & $\begin{array}{c}46(85.2) \\
8(14.8) \\
54(100.0) \\
52(96.3) \\
48(88.9) \\
31(57.4)\end{array}$ & $\begin{array}{c}40(71.4) \\
16(28.6) \\
56(100.0) \\
55(98.2) \\
45(80.4) \\
18(32.1)\end{array}$ & $\begin{array}{l}0.29 \\
\mathbf{0 . 0 2}\end{array}$ \\
\hline
\end{tabular}

MPNST: malignant peripheral nerve sheath tumor.

between both patient groups (6 months [95\% CI 4-8 months] for M-STS patients vs. 11 months [95\% CI 9-13 months] for LA-STS patients, $p=0.185$, data not shown).

For patients with nonmetastatic disease (LA-STS), median PFS was independent of age ( $>50$ years vs. $\leq 50$ years), sex, and treatment line (second-line treatment vs. >second- line treatment; data not shown). In univariate analysis, grading, histologic subtype (lipo/leiomyosarcoma vs. other subentities), type of progression (primary refractory disease vs. progression after response to prior chemotherapy), response to ICE + RHT (SD/PR/CR vs. PD), and surgery significantly influenced progression-free survival. In 
TABLE 2: ICE + RHT treatment characteristics.

\begin{tabular}{|c|c|c|}
\hline & $\begin{array}{l}\text { Locally advanced disease } \\
\quad(\text { LA-STS, } n=54)\end{array}$ & $\begin{array}{l}\text { Metastatic disease } \\
(\mathrm{M}-\mathrm{STS}, n=56)\end{array}$ \\
\hline & Median (range) & Median (range) \\
\hline ICE cycles & $4(1-8)$ & $4(1-8)$ \\
\hline \multirow[t]{2}{*}{ RHT treatments } & $8(2-16)$ & $6(1-16)$ \\
\hline & No. of patients (\%) & No. of patients (\%) \\
\hline Radiotherapy & $6(11.1)$ & $6(10.7)$ \\
\hline Surgery & $31(57.4)$ & $15(26.8)$ \\
\hline Surgery prior to start of ICE $+\mathrm{RHT}$ & $10(18.5)$ & $1(1.8)$ \\
\hline R0 & $7(13.0)$ & $5(8.8)$ \\
\hline $\mathrm{R} 1$ & $10(18.5)$ & $4(7.2)$ \\
\hline $\mathrm{R} 2$ & $10(18.5)$ & $4(7.2)$ \\
\hline $\mathrm{Rx}$ & $4(7.4)$ & $2(3.6)$ \\
\hline Including metastasectomy & & $8(14.3)$ \\
\hline Excluding metastasectomy & & $7(12.5)$ \\
\hline
\end{tabular}

R0, complete resection of the tumor, with no microscopic evidence of tumor infiltration of resection margins; R1, complete macroscopic resection of the tumor, with microscopic evidence of tumor infiltration of resection margins; R2, incomplete resection of the tumor, with macroscopic tumor burden remaining in situ; $\mathrm{Rx}$, resection with unknown resection margins.

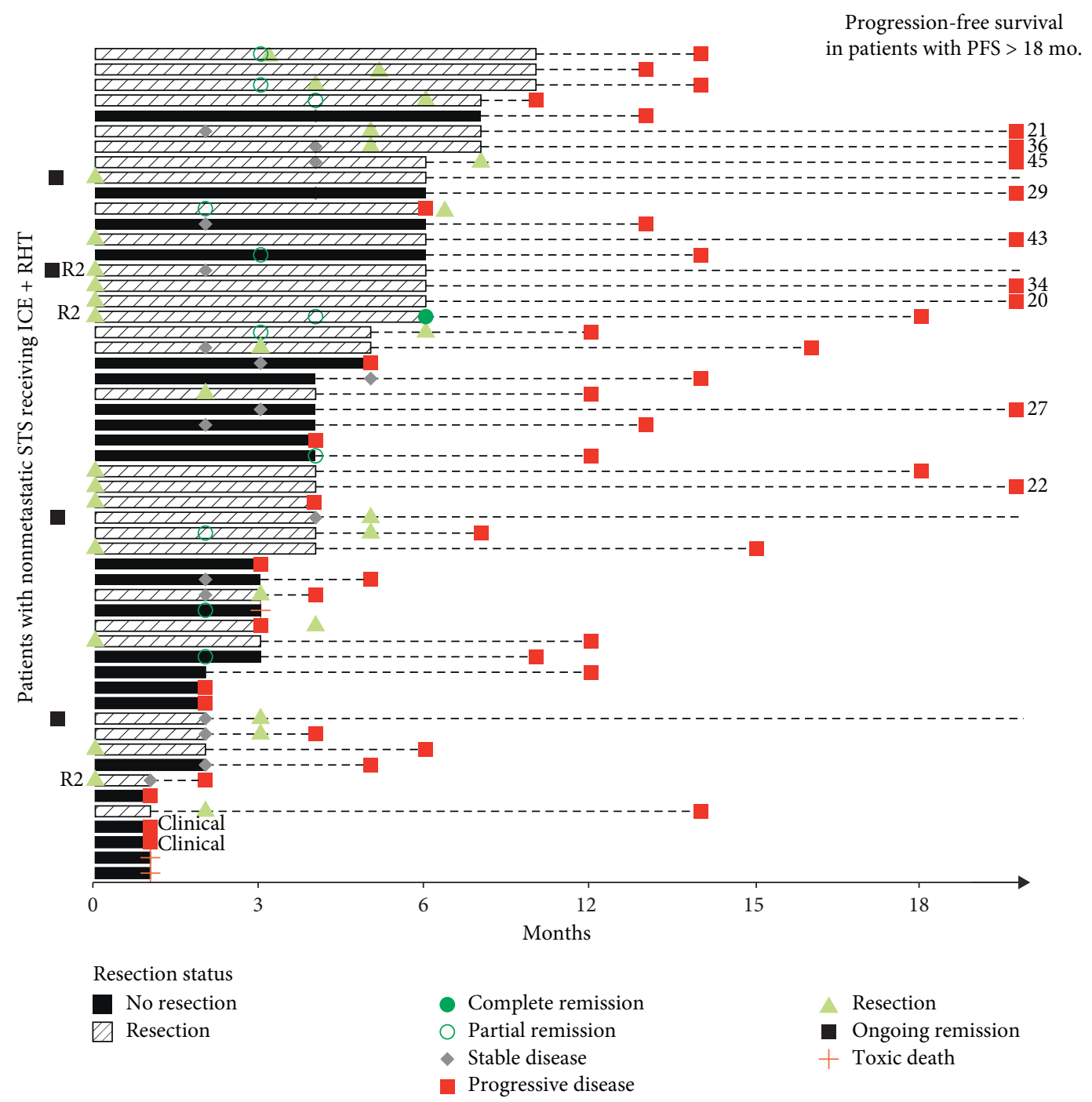

Figure 1: Swimmer plot depicting the clinical course of LA-STS patients $(n=54)$. Bars represent the treatment duration of ICE + RHT (range: 1-8 months). Results of radiographic assessments and other major clinical events are represented by different symbols, as depicted on the bottom. mo., months and PFS, progression-free survival. 
TABLE 3: Radiographic response to ICE + RHT treatment. For patients receiving tumor resection during or directly after ICE + RHT, radiographic response is given for the latest time point prior to resection. Percentages of CR, PR, NC and PD are given as proportions of patients with measurable disease.

\begin{tabular}{|c|c|c|}
\hline & $\begin{array}{c}\text { Locally advanced disease } \\
(\text { LA-STS, } n=54)\end{array}$ & $\begin{array}{c}\text { Metastatic disease } \\
(\mathrm{M}-\mathrm{STS}, n=56)\end{array}$ \\
\hline & No. (\%) & No. (\%) \\
\hline No measurable disease & $10(18.5)$ & $1(1.8)$ \\
\hline Measurable disease & $44(81.5)$ & $55(98.2)$ \\
\hline $\mathrm{CR}$ & $1(2.3)$ & $0(0.0)$ \\
\hline PR & $8(18.2)$ & $6(10.9)$ \\
\hline SD & $17(38.5)$ & $20(36.4)$ \\
\hline $\mathrm{PD}$ & $9(20.5)$ & $24(43.6)$ \\
\hline Could not be evaluated & $9(20.5)$ & $5(9.1)$ \\
\hline Objective response & $9(20.5)$ & $6(10.9)$ \\
\hline Disease control & $26(59.1)$ & $26(47.3)$ \\
\hline
\end{tabular}

$\mathrm{CR}$, complete response; $\mathrm{SD}$, stable disease; $\mathrm{PD}$, progressive disease; $\mathrm{PR}$, partial response.

TABLE 4: Progression-free survival rates 3 and 6 months after initiation of ICE + RHT.

\begin{tabular}{lcc}
\hline Patient subpopulation & PFR after 3 months (95\% CI) & PFR after 6 months (95\% CI) \\
\hline Patients with nonmetastatic disease $(n=54$, LA-STS) & $82 \%(72-93 \%)$ & $67 \%(56-82 \%)$ \\
Patients with metastatic disease $(n=56$, M-STS) & $54 \%(42-68 \%)$ & $23 \%(14-37 \%)$ \\
Patients with metastatic disease without surgical treatment $(n=41)$ & $41 \%(29-60 \%)$ & $20 \%(10-36 \%)$ \\
\hline
\end{tabular}

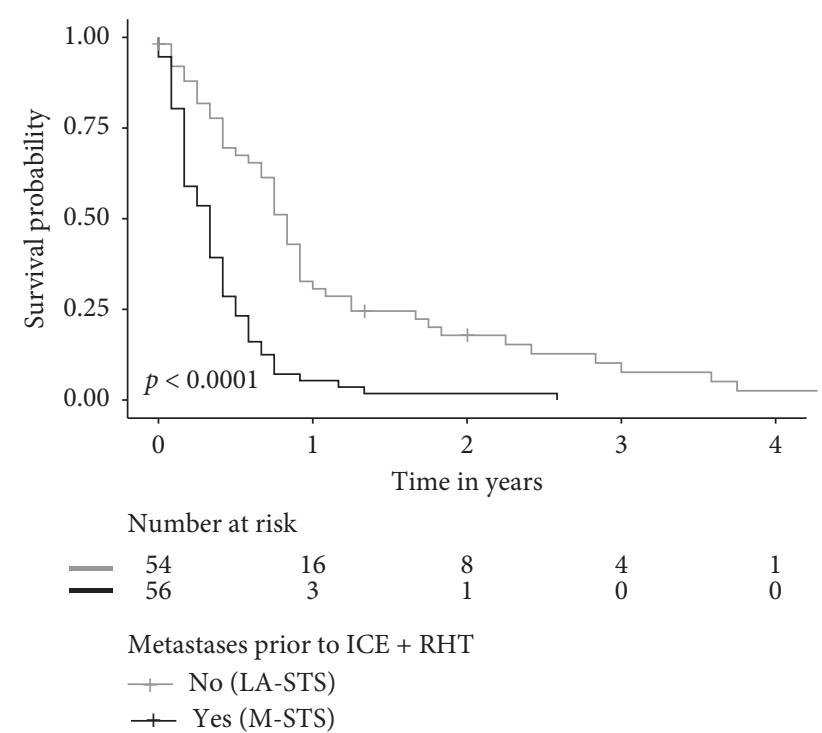

Figure 2: Kaplan-Meier estimates of progression-free survival for patients with $(n=56, \mathrm{M}-\mathrm{STS})$ and without $(n=54$, LA-STS $)$ metastases prior to initiation of ICE + RHT. The $p$ value was assessed by the log-rank test.

multivariate analysis, grading, type of relapse, and response to therapy remained of significant influence (Supplementary Table 1).

For M-STS patients, response to ICE + RHT (SD/PR/CR vs. PD) was the only factor significantly influencing PFS in univariate $(p<0.0001)$ and multivariate analyses $(p<0.001$, data not shown).

3.4.2. Overall Survival. Median overall survival for the LASTS population was 26 months (95\% CI 18-31 months). Patients with metastatic disease (M-STS) had a significantly shorter overall survival (12 months, 95\% CI 10-17 months, $p=0.002$; data not shown). Factors significantly influencing overall survival in the univariate analysis for LA-STS patients were surgical resection of the tumor, type of progression (primary refractory disease vs. progression after response to prior chemotherapy), and response to ICE + RHT (SD/PR/ CR vs. PD, 27 vs. 9 months, $p=0.039$, Figure 3). The latter remained the only independent factor influencing overall survival in the multivariate analysis (Table 5).

Similar to PFS, response to ICE + RHT (SD/PR/CR vs. $\mathrm{PD})$ remained the only factor significantly influencing $\mathrm{OS}$ in the univariate $(p<0.0001)$ and multivariate analysis $(p=0.002)$ for M-STS patients (Supplementary Table 2).

3.5. Hematological Toxicity, Dose Reductions, and Infectious Complications. Hematological toxicity was common after treatment with ICE + RHT. Of all treated patients, almost two-thirds showed grade III or IV toxicity according to the CTCAE V4.03 criteria (Table 6). Therapy-induced neutropenia lead to infectious complications with fever in 27 of the 110 treated patients $(24.5 \% ; 15 / 54$ in LA-STS and 12/56 in M-STS). Three LA-STS patients died due to therapyrelated infections $(6 \%$ of LA-STS patients or $3 \%$ of all patients, Table 6).

Dose reductions were frequent. In the majority of patients, applying a full-dosed ICE cycle was never deemed feasible. Further dose reductions were needed in more than $50 \%$ of all patients, including patients that never received a full-dosed ICE cycle. Relative dose intensities are reported in Supplementary Figure 2.

\section{Discussion}

Prognosis of patients with STS has shown little improvement over the past decades [14]. Wide tumor resection remains 


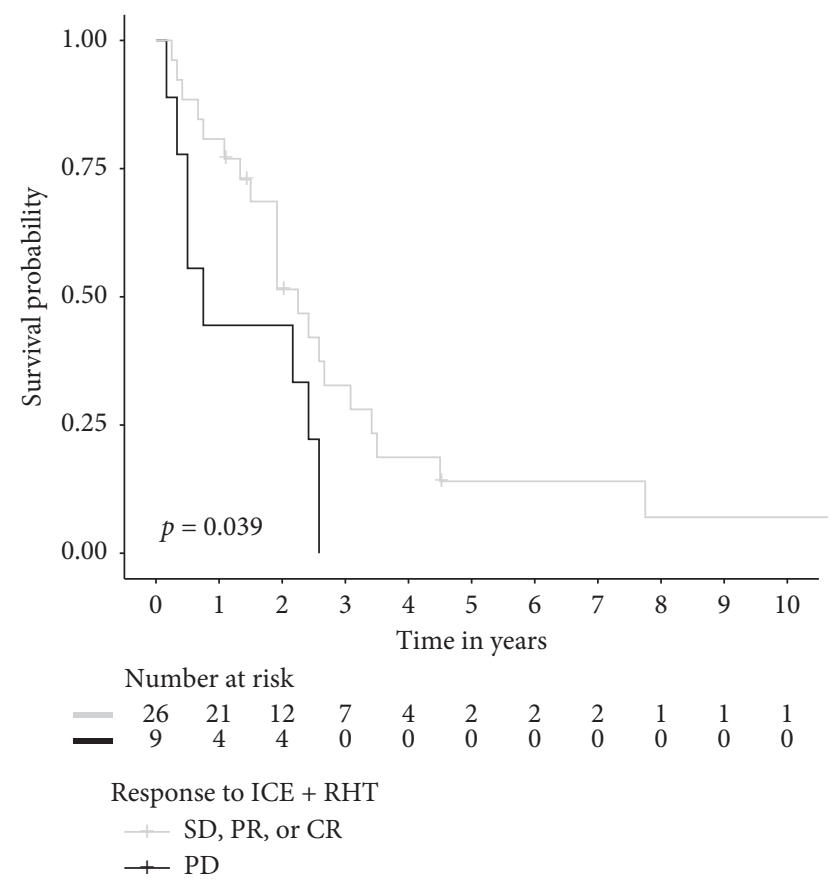

FIGURE 3: Kaplan-Meier estimates of overall survival for LA-STS patients with disease stabilization or response (SD/PR/CR, $n=26$ ) and progressive disease (PD, $n=9$ ) as the best radiographic response to ICE + RHT. CR, complete response; SD, stable disease; $\mathrm{PD}$, progressive disease; and $\mathrm{PR}$, partial remission. The $p$ value was assessed by the log-rank test.

standard of care. In patients with high-risk disease (G2/3, tumor diameter $\geq 5 \mathrm{~cm}$, location deep to the fascia), treatment is regularly intensified by radiotherapy and anthracycline-containing chemotherapy.

However, relapse after resection, even after intensified pretreatment, occurs frequently, particularly in patients with retroperitoneal or visceral location of their tumor. When relapse is limited to locoregional recurrence of the tumor, a curative approach is appropriate. Even with oligometastatic disease, metastasectomy should be considered, as metastatic spread does not preclude durable remissions after successful resection [22]. However, no evidence-based treatment strategy has been outlined for patients in this situation so far [1], and there is a clear medical need to improve the outcome.

Here, we report results of a multimodal therapy approach for patients with failure of a previous anthracyclinecontaining chemotherapy. The multimodal approach included RHT to maximize the local efficacy of chemotherapy. RHT, when applied in parallel, improves response rates of chemotherapy in high-risk STS, compared with chemotherapy alone [10], and might therefore facilitate resection in otherwise nonresectable STS. ICE, a polychemotherapy regimen reportedly active in smaller series of STS patients $[23,24]$, was used. To our knowledge, our analysis is the first to report results of an intensified perioperative chemotherapy approach in the context of relapsed or refractory STS. In addition to the LA-STS and M-STS general analyses, we also separately analyzed the outcomes of a subcohort with metastatic STS (M-STS), treated with ICE + RHT at our institution over the past years to estimate the efficacy of the treatment. All patients in this subcohort had predominant local relapse and therefore received chemotherapy in combination with RHT. Nevertheless, the M-STS population closely resembled the patient populations used by Glabbeke et al. to establish reference values to determine efficacy in anthracycline-refractory STS [19]. Estimation of PFR three months after initiation of therapy with ICE + RHT in the M-STS subcohort showed ongoing response to treatment or progression arrest in $54 \%$ of the patients, indicative of an active treatment regimen (determined to induce a PFR $\geq 40 \%$ three months after initiation of treatment) per the criteria proposed by Glabbeke et al. PFR after three months in patients with metastatic disease who did not receive tumor resection also met the criteria of activity (41\%, Table 4$)$.

Response rates were higher in LA-STS patients than in the M-STS population ( $21 \%$ vs. $11 \%$, Table 2$)$, comparing favorably with single-agent therapies applied in anthracycline-refractory STS $[11,12,25]$. Interestingly, progressionfree survival was shorter in M-STS patients (4 vs. 10 months, Figure 2) due to progression of metastasis. Local progression-free survival was not significantly different between LASTS and M-STS patients, hinting at the contribution of RHT treatment to an improved disease control.

Response to ICE + RHT treatment facilitated resection in a subgroup of patients, which accounted for 7\% of the LASTS population without metastases and 9\% of the M-STS population. Tumor resection is a contributor with borderline significance in multivariate analyses for progression-free and overall survival in the LA-STS subcohort, and ICE + RHT might therefore lead to improved survival for patients with otherwise dismal prognosis due to the impossibility of sufficient tumor resection. The strongest evidence for the favorable activity of ICE + RHT, however, derives from the observation that disease stabilization, or CR, or PR with ICE + RHT was associated with improved OS in the univariate analysis (Figure 3), which remained the only significant prognostic factor for improved overall survival in the multivariate analysis (Table 5) for both LA-STS and M-STS patients. Recently, Grünwald et al. demonstrated that the absence of disease progression is the main factor predicting therapeutic efficacy of anthracycline-based chemotherapy in patients with advanced STS [26]. This is in line with our findings, highlighting the major importance of disease control for improved outcome in STS.

Response kinetics show that the best response is usually achieved within two to four cycles of treatment, as only one patient with a PR after four cycles achieved a deeper remission (CR) after six cycles of chemotherapy within the LASTS subcohort. In contrast, progression of disease during ongoing ICE + RHT treatment in patients with initially stable or responding STS occurred in five patients in the LASTS subcohort and eleven patients in the M-STS subcohort. These observations hint at the necessity of early re-resection of the tumor after two to four cycles of therapy.

As progression-free and overall survival were independent of STS subentities (lipo-/leiomyosarcoma vs. other subentities) in the multivariate analysis, ICE + RHT can be 
TABLE 5: Prognostic factors for overall survival for LA-STS patients. $p$ values were calculated using the log-rank test for univariate analysis. For multivariate analysis using a Cox model, only parameters with a $p<0.10$ in univariate analyses were considered.

\begin{tabular}{|c|c|c|c|c|c|}
\hline \multirow[b]{2}{*}{ Parameter } & \multirow[b]{2}{*}{$N$} & \multicolumn{2}{|c|}{ Univariate analysis } & \multicolumn{2}{|c|}{ Cox model } \\
\hline & & $\begin{array}{l}\text { Median OS, mo. } \\
\quad(95 \% \mathrm{CI})\end{array}$ & $p$ & $\begin{array}{c}\mathrm{HR} \\
(95 \% \mathrm{CI})\end{array}$ & $p$ \\
\hline Age & & & 0.46 & & \\
\hline$>50$ years & 38 & $26(18-40)$ & & & \\
\hline$\leq 50$ years & 16 & $23(10-41)$ & & & \\
\hline Sex & & & 0.57 & & \\
\hline Male & 31 & $28(13-41)$ & & & \\
\hline Female & 23 & $23(18-93)$ & & & \\
\hline Treatment line & & & 0.31 & & \\
\hline 2nd line & 46 & $26(18-37)$ & & & \\
\hline$>2$ nd line & 8 & $20(10-\mathrm{NA})$ & & & \\
\hline Grading & & & 0.12 & & \\
\hline 3 & 32 & $23(16-31)$ & & & \\
\hline 2 & 22 & $31(28-\mathrm{NA})$ & & & \\
\hline Histologic subentity & & & 0.23 & & \\
\hline Lipo-/leiomyosarcoma & 28 & $29(13-93)$ & & & \\
\hline Non-lipo-/leiomyosarcoma & 26 & $23(18-37)$ & & & \\
\hline Surgery & & & 0.014 & $0.52(0.25-1.10)$ & 0.088 \\
\hline Yes & 31 & $18(8-31)$ & & & \\
\hline No & 23 & $28(23-93)$ & & & \\
\hline $\begin{array}{l}\text { Response/disease stabilization as the best radiographic } \\
\text { response with ICE + RHT }\end{array}$ & & & 0.039 & $0.42(0.18-0.96)$ & 0.041 \\
\hline Yes & 26 & $27(23-41)$ & & & \\
\hline No & 9 & $9(6-\mathrm{NA})$ & & & \\
\hline Type of progression & & & $<0.001$ & $0.69(0.33-1.45)$ & 0.332 \\
\hline Progression after response to prior chemotherapy & 32 & $31(23-94)$ & & & \\
\hline Primary refractory disease & 22 & $13(9-31)$ & & & \\
\hline
\end{tabular}

HR, hazard ratio; mo., months; NA, not assessable; OS, overall survival.

TABLE 6: Toxicity, according to the CTCAE 4.03 criteria, and dose reductions of ICE + RHT. Dose information was available for 106 patients (53 with locally advanced and 53 with metastatic disease).

\begin{tabular}{lcc}
\hline & $\begin{array}{c}\text { Locally advanced disease } \\
\text { (LA-STS, } n=54)\end{array}$ & $\begin{array}{c}\text { Metastatic disease } \\
(\mathrm{M}-\mathrm{STS}, n=56)\end{array}$ \\
\hline Hematological toxicity & $\begin{array}{c}\text { No. of patients }(\%) \\
11(20.4)\end{array}$ & $\begin{array}{c}\text { No. of patients }(\%) \\
13(23.2)\end{array}$ \\
Grade III & $25(46.3)$ & $20(35.7)$ \\
Grade IV & $15(27.8)$ & $12(21.4)$ \\
Fever in cytopenia & $3(5.6)$ & $0(0.0)$ \\
Therapy-related deaths & $30(55.6)$ & $31(55.4)$ \\
ICE chemotherapy doses & $23(42.6)$ & $32(57.1)$ \\
Patients never receiving full dose & $1(1.8)$ & $3(5.3)$ \\
Further dose reductions needed (compared to dose of first applied cycle) & \\
No information on dosing available &
\end{tabular}

considered as salvage therapy in a wide range of STS histologies (Table 5 and Supplementary Table 1).

However, this favorable antitumor activity is associated with relevant toxicity. Grade III/IV hematological toxicity was observed in more than $60 \%$ of all patients, despite dose reductions in more than $50 \%$ of all patients. Neutropenic fever frequently occurred and lead to three sepsis-related deaths. Compared with other chemotherapeutic regimens used in the second-line treatment of STS, hematologic toxicity and infectious complications are common with ICE + RHT [11, 12, 27-29]. We therefore adapted the starting dose of ICE + RHT and recommend a starting dose of $75 \%$ of the originally intended dose (cumulative doses per cycle: ifosfamide $4.5 \mathrm{~g} / \mathrm{m}^{2}$, carboplatin $300 \mathrm{mg} / \mathrm{m}^{2}$, and etoposide $450 \mathrm{mg} / \mathrm{m}^{2}$ ).

\section{Conclusions}

$\mathrm{ICE}+\mathrm{RHT}$ is an active salvage therapy regimen in the treatment of STS patients after the failure of an anthracycline-containing pretreatment. In patients with nonresectable localized relapse or with progression during anthracycline-based chemotherapy, ICE + RHT leads to disease stabilization in a significant proportion of patients and can facilitate resection in a subgroup of patients. 
The use of ICE + RHT in patients with locally advanced relapse or progression under anthracycline-based pretreatment should therefore be evaluated in prospective clinical trials. Patients planned for re-resection should receive no more than four cycles of treatment, as patients without radiographic evidence of response are not expected to respond later on, and patients with initial stable or responding STS may have subsequent disease progression. If resectability cannot be reached, treatment with ICE + RHT should be continued nevertheless, as disease stabilization and response are associated with improved OS in these patients with otherwise dismal prognosis.

\section{Data Availability}

The data used to support the findings of this study are available from the corresponding author upon request.

\section{Conflicts of Interest}

S.A.R. received travel support and honoraria from Pyrexar and Medtherm. L.H.L. received research and travel support from Dr. Sennewald Medizintechnik, travel support from PharmaMar, and honoraria from Novartis, Lilly, Eisai, and EL Medconsult. All other authors declare that there are no conflicts of interest regarding the publication of this article.

\section{Supplementary Materials}

Supplementary Figure 1: selected patient populations. Abbreviations: STS, soft-tissue sarcoma. Supplementary Figure 2: relative dose intensity of applied ICE cycles. Fulldosed ICE (as described in the patients and methods paragraph) over four days was considered $100 \%$. Supplementary Table 1: prognostic factors for progression-free survival for LA-STS patients. $p$ values were calculated using the log-rank test for univariate analysis. For multivariate analysis using a Cox model, only parameters with a $p<0.10$ in univariate analyses were considered. Abbreviations: HR, hazard ratio; mo, months; NA, not assessable; OS, overall survival. Supplementary Table 2: prognostic factors for overall survival for M-STS patients. $p$ values were calculated using the $\log$-rank test for univariate analysis. For multivariate analysis using a Cox model, only parameters with a $p<0.10$ in univariate analyses were considered. Abbreviations: HR, hazard ratio; mo, months; NA, not assessable; OS, overall survival. (Supplementary Materials)

\section{References}

[1] P. Casali, N. Abecassis, S. Bauer et al., "Soft tissue and visceral sarcomas: ESMO-EURACAN clinical practice guidelines for diagnosis, treatment and follow-up," Annals of Oncology, vol. 29, no. 4, pp. 51-67, 2018.

[2] A. Stojadinovic, D. H. Y. Leung, A. Hoos, D. P. Jaques, J. J. Lewis, and M. F. Brennan, "Analysis of the prognostic significance of microscopic margins in 2,084 localized primary adult soft tissue sarcomas," Annals of Surgery, vol. 235, no. 3, pp. 424-434, 2002.

[3] A. Gronchi, R. Miceli, M. Fiore et al., "Extremity soft tissue sarcoma: adding to the prognostic meaning of local failure,"
Annals of Surgical Oncology, vol. 14, no. 5, pp. 1583-1590, 2007.

[4] D. J. Biau, P. C. Ferguson, P. Chung et al., "Local recurrence of localized soft tissue sarcoma," Cancer, vol. 118, no. 23, pp. 5867-5877, 2012.

[5] A. Italiano, A. Le Cesne, J. Mendiboure et al., "Prognostic factors and impact of adjuvant treatments on local and metastatic relapse of soft-tissue sarcoma patients in the competing risks setting," Cancer, vol. 120, no. 21, pp. 3361-3369, 2014.

[6] V. Kainhofer, M. A. Smolle, J. Szkandera et al., "The width of resection margins influences local recurrence in soft tissue sarcoma patients," European Journal of Surgical Oncology (EJSO), vol. 42, no. 6, pp. 899-906, 2016.

[7] R. D. Issels, L. H. Lindner, J. Verweij et al., "Neo-adjuvant chemotherapy alone or with regional hyperthermia for localised high-risk soft-tissue sarcoma: a randomised phase 3 multicentre study," The Lancet Oncology, vol. 11, no. 6, pp. 561-570, 2010.

[8] S. Abatzoglou, R. E. Turcotte, A. Adoubali, M. H. Isler, and D. Roberge, "Local recurrence after initial multidisciplinary management of soft tissue sarcoma: is there a way out?," Clinical Orthopaedics and Related Research, vol. 468, no. 11, pp. 3012-3018, 2010.

[9] A. Daigeler, I. Zmarsly, T. Hirsch et al., "Long-term outcome after local recurrence of soft tissue sarcoma: a retrospective analysis of factors predictive of survival in 135 patients with locally recurrent soft tissue sarcoma," British Journal of Cancer, vol. 110, no. 6, pp. 1456-1464, 2014.

[10] R. D. Issels, L. H. Lindner, J. Verweij et al., "Effect of neoadjuvant chemotherapy plus regional hyperthermia on longterm outcomes among patients with localized high-risk soft tissue sarcoma: the EORTC 62961-ESHO 95 randomized clinical Trial," JAMA Oncology, vol. 4, no. 4, p. 483, 2018.

[11] P. Schöffski, S. Chawla, R. G. Maki et al., "Eribulin versus dacarbazine in previously treated patients with advanced liposarcoma or leiomyosarcoma: a randomised, open-label, multicentre, phase 3 trial," The Lancet, vol. 387, no. 10028, pp. 1629-1637, 2016.

[12] W. T. van der Graaf, J.-Y. Blay, S. P. Chawla et al., "Pazopanib for metastatic soft-tissue sarcoma (PALETTE): a randomised, double-blind, placebo-controlled phase 3 trial," The Lancet, vol. 379, no. 9829, pp. 1879-1886, 2012.

[13] G. D. Demetri, S. P. Chawla, M. von Mehren et al., "Efficacy and safety of trabectedin in patients with advanced or metastatic liposarcoma or leiomyosarcoma after failure of prior anthracyclines and ifosfamide: results of a randomized phase II study of two different schedules," Journal of Clinical Oncology, vol. 27, no. 25, pp. 4188-4196, 2009.

[14] P. Schöffski, J. Cornillie, A. Wozniak, H. Li, and D. Hompes, "Soft tissue sarcoma: an update on systemic treatment options for patients with advanced disease," Oncology Research and Treatment, vol. 37, no. 6, pp. 355-362, 2014.

[15] V. Y. Jo and C. D. M. Fletcher, "WHO classification of soft tissue tumours: an update based on the 2013 (4th) edition," Pathology, vol. 46, no. 2, pp. 95-104, 2014.

[16] G. Bruggmoser, S. Bauchowitz, R. Canters et al., "Guideline for the clinical application, documentation and analysis of clinical studies for regional deep hyperthermia: quality management in regional deep hyperthermia," Strahlentherapie und Onkologie, vol. 188, no. 2, pp. 198-211, 2012.

[17] E. A. Eisenhauer, P. Therasse, J. Bogaerts et al., "New response evaluation criteria in solid tumours: revised RECIST guideline (version 1.1)," European Journal of Cancer, vol. 45, no. 2, pp. 228-247, 2009. 
[18] National Institutes of Health, National Cancer Institute: Common Terminology Criteria For Adverse Events (CTCAE), V4.03, 2009, National Institutes of Health, Bethesda, MA, USA, 2015.

[19] V. M. Glabbeke, J. Verweij, I. Judson, and O. S. Nielsen, "Progression-free rate as the principal end-point for phase II trials in soft-tissue sarcomas," European Journal of Cancer, vol. 38 , no. 4, pp. 543-549, 2002.

[20] M. Greenwood, "The natural duration of cancer: reports on public health and medical subjects," Her Majesty's Stationery Office, vol. 33, pp. 1-26, 1926.

[21] T. Therneau, A Package for Survival Analysis in S, Version 2.35, 2019, https://CRAN.R-project.org/package=survival.

[22] K. G. Billingsley, M. E. Burt, E. Jara et al., "Pulmonary metastases from soft tissue sarcoma: analysis of patterns of diseases and postmetastasis survival," Annals of Surgery, vol. 229, no. 5, pp. 602-610, 1999.

[23] K. K. Fields, P. E. Zorsky, J. W. Hiemenz, L. E. Kronish, and G. J. Elfenbein, "Ifosfamide, carboplatin, and etoposide: a new regimen with a broad spectrum of activity," Journal of Clinical Oncology, vol. 12, no. 3, pp. 544-552, 1994.

[24] M. Fiegl, M. Schlemmer, C.-M. Wendtner, S. Abdel-Rahman, W. Fahn, and R. D. Issels, "Ifosfamide, carboplatin and etoposide (ICE) as second-line regimen alone and in combination with regional hyperthermia is active in chemo-pretreated advanced soft tissue sarcoma of adults," International Journal of Hyperthermia, vol. 20, no. 6, pp. 661-670, 2004.

[25] A. Yovine, M. Riofrio, J. Y. Blay et al., "Phase II study of ecteinascidin-743 in advanced pretreated soft tissue sarcoma patients," Journal of Clinical Oncology, vol. 22, no. 5, pp. 890-899, 2004.

[26] V. Grünwald, S. Litière, R. Young et al., "Absence of progression, not extent of tumour shrinkage, defines prognosis in soft-tissue sarcoma - an analysis of the EORTC 62012 study of the EORTC STBSG," European Journal of Cancer, vol. 64, pp. 44-51, 2016.

[27] A. Kawai, N. Araki, H. Sugiura et al., "Trabectedin monotherapy after standard chemotherapy versus best supportive care in patients with advanced, translocation-related sarcoma: a randomised, open-label, phase 2 study," The Lancet Oncology, vol. 16, no. 4, pp. 406-416, 2015.

[28] M. L. Hensley, J. A. Blessing, R. Mannel, and P. G. Rose, "Fixed-dose rate gemcitabine plus docetaxel as first-line therapy for metastatic uterine leiomyosarcoma: a gynecologic oncology group phase II trial," Gynecologic Oncology, vol. 109, no. 3, pp. 329-334, 2008.

[29] J.-Y. Blay, Z. Pápai, A. W. Tolcher et al., "Ombrabulin plus cisplatin versus placebo plus cisplatin in patients with advanced soft-tissue sarcomas after failure of anthracycline and ifosfamide chemotherapy: a randomised, double-blind, placebo-controlled, phase 3 trial," The Lancet Oncology, vol. 16, no. 5, pp. 531-540, 2015. 